\title{
Estratégia de Saúde Mental e Atenção Psicossocial para Afetados da Boate Kiss
}

\author{
Débora da Silva Noal \\ Universidade de Brasília, DF, Brasil. \\ Ana Cecília Andrade de Moraes Weintraub \\ Universidade de São Paulo, SP, Brasil. \\ Károl Veiga Cabral \\ Universitat Rovira i Virgili, Espanha. \\ Maria Luiza Leal Pacheco \\ Pontifícia Universidade Católica do Rio Grande do Sul, \\ RS, Brasil. \\ Letícia Nolasco Vicente \\ Instituto Sedes Sapientiae, SP, Brasil. \\ Sandra Maria Sales Fagundes \\ Universidade Federal do Rio Grande do Sul, RS, Brasil. \\ Ana Carolina Rios Simoni \\ Universidade Federal do Rio Grande do Sul, RS, Brasil. \\ Regina Lucia Sucupira Pedroza \\ Universidade de Brasília, DF, Brasil. \\ Lucia Helena Cavasin Zabotto Pulino \\ Universidade de Brasília, DF, Brasil.
}

Resumo: Eventos críticos (EC) são eventos que dão início a uma cadeia de incidentes, resultando em uma situação de desastre, a menos que um sistema de segurança interfira para evitá-lo ou minimizá-lo. Este artigo apresenta um relato de experiência durante um EC em que psicólogas elaboraram a estratégia de gestão da atenção psicossocial e saúde mental decorrente do incêndio na boate Kiss, ocorrido em 2013, na cidade de Santa Maria (RS). O objetivo deste relato de experiência é apresentar e analisar a estratégia elaborada de apoio psicossocial e de saúde mental. Fazendo uso de métodos qualitativos, iniciamos a discussão descrevendo a rede pública de Atenção Psicossocial do município na época em que ocorreu o incêndio. Na sequência, delineia-se uma análise descritiva das medidas tomadas nas primeiras 24 horas após esse evento e, finalmente, apresentam-se as estratégias elaboradas para os meses subsequentes. Para esse relato foram utilizados dados e referências do Sistema Único de Saúde e do Sistema Nacional de Proteção e Defesa Civil, contemplando o panorama nacional no momento da intervenção. Dentre os resultados destacam-se a imprescindibilidade do trabalho articulado no nível loco-regional e o delineamento da avaliação sistemática envolvendo os três entes federados. Atividades e ações elaboradas como resposta foram estruturadas de forma condizente com as necessidades psicossociais do público-alvo, bem como com as diretrizes e políticas (inter) nacionais, enfocando a criação de uma estratégia articulada e sustentável em um curto e médio prazo e visando o bem-estar psicossocial dos afetados.

Palavras-chave: Intervenção na Crise, Serviços de Saúde Mental. Saúde Pública. Intervenção Psicossocial. 


\title{
Mental Health and Psychosocial Care Strategy for Affected from Kiss Nightclub
}

\begin{abstract}
Critical incidents (CI) are events that trigger a series of other incidents resulting in a disaster situation, unless a security system takes place in order to avoid or minimize it. This article presents an experience reported by a group of psychologists responsible for a specific CI: the fire occurred at Kiss nightclub in Santa Maria/RS in 2013, that resulted in the death of 242 people and left many others injured. The objective of this text is to describe and analyze the psychosocial support and mental health strategy elaborated during that event. First, the article shows how Santa Maria's public mental health network was set at the time of the event. Then, it lists and analyzes the measures adopted in the first 24 hours after the CI. Finally, it discusses the strategy created for the following weeks and months. Data from the Brazilian Unified Health System (SUS) and the National System of Protection and Civil Defense were also used, in order to assist the intervention analysis. The activities proposed at the time not only appeared to be in agreement with the minimum psychosocial needs of the target population, but also aligned with national and international guidelines, that value articulated and sustainable, short and midterm strategies, capable of contributing with the well-being of the citizens.
\end{abstract}

Keywords: Crisis Intervention, Mental Health Services, Public Health, Psychosocial Intervention.

\section{Estrategia de Salud Mental y Atención Psicosocial para Afectados de la Discoteca Kiss}

Resumen: Los incidentes críticos (IC) son eventos que estimulan una cadena de otros incidentes que resultan en una situación de desastre, a menos que un sistema de seguridad se ponga en marcha con el fin de evitar o reducir al mínimo el desastre. Este artículo tiene como objetivo presentar un relato de una experiencia de un grupo de psicólogas que trabajó en una situación de incidente crítico (IC) - el incendio en la discoteca Kiss en Santa María, RS en 2013. El objetivo del texto es presentar la estrategia de apoyo psicosocial y de salud mental elaborado durante ese evento. Con el fin de hacer eso, presentamos, en primer lugar, la red de salud mental pública que existía en Santa María en el momento en que ocurrió el incendio. En segundo lugar, mostramos las medidas adoptadas en las primeras 24 horas después del IC. Finalmente, se discute la estrategia elaborada para las siguientes semanas y meses. Los datos del sistema de salud pública y el Sistema Nacional de Protección y Defensa Civil también se utilizaron para ayudar a analizar la intervención. Los resultados destacan el carácter indispensable de la labor conjunta del nivel local y regional y el diseño de una evaluación sistemática entre los tres entes federales. Las actividades propuestas en el momento estaban en línea con las necesidades psicosociales de la población objetivo y con las directrices nacionales e internacionales, centrándose en la creación de una estrategia articulada y sostenible en el corto y mediano plazo para contribuir con el bienestar de los ciudadanos.

Palabras clave: Intervención en Crisis, Servicios de Salud Mental, Salud Pública, Intervención Psicosocial. 


\section{Introdução}

Este artigo analisa os primeiros três meses da estratégia de Atenção Psicossocial (AP) e Saúde Mental (SM) criada em resposta ao incêndio ocorrido na Boate Kiss no município de Santa Maria, RS, em 27 de janeiro de 2013. Essa reflexão parte da experiência de seis das autoras deste artigo como membros da equipe que conformou a primeira resposta ao evento a partir do lugar que ocupavam enquanto psicólogas voluntárias na estratégia de Saúde Mental, gestoras da Secretaria Estadual de Saúde do Rio Grande do Sul, membros de uma organização internacional especializada em emergências e, também, em um momento posterior à fase aguda, participação como consultora do Ministério da Saúde responsável pela primeira avaliação da resposta. Dessa forma, o objetivo deste relato de experiência é apresentar e analisar uma intervenção que ofereceu cuidados psicossociais e de saúde mental aos afetados pelo Evento Crítico (EC).

Como contribuição ao campo da Psicologia na Gestão Integral de Riscos e de Desastres (GRD), a publicização deste relato de experiência visa disseminar os procedimentos de elaboração e acompanhamento de uma intervenção psicossocial em contexto de $\mathrm{EC}$, a fim de que a atenção às pessoas que vivenciam o EC seja conhecida, difundida e aprimorada. Considerando a perspectiva cultural, buscamos fortalecer habilidades de enfrentamento em eventos desta natureza (Paranhos, \& Werlang, 2015; Tol et al., 2011; Van Ommeren, Saxena, \& Saraceno, 2005).

\section{Contextualizando o Incêndio na Boate Kiss}

$\mathrm{O}$ incêndio na boate Kiss causou a morte de 242 pessoas e deixou outras 680 feridas (Noal, Vicente, \& Weintraub, 2016). As vítimas foram majoritariamente jovens universitários que frequentavam a boate em virtude de uma festa promovida por alunos das faculdades em que estudavam. O incêndio foi um evento amplamente noticiado e que gerou intensa comoção nacional e internacional. A classificação de um acontecimento dessa natureza como "desastre", "evento crítico", "catástrofe" ou termos correlatos é tema controverso e de intenso debate histórico, institucional e sociopolítico. No Brasil, vige atualmente a Instrução Normativa no 1 (Brasil, 2012a), baseada na Lei ${ }^{\circ}$ 12.608/2012 (Brasil, 2012b), na qual se encontra uma definição corrente desses diferentes termos com base numa análise do tipo de desastre (Nível I ou II), das características, dos danos (humanos, materiais e/ou ambientais) e prejuízos para o território que um determinado evento acarreta.

O incêndio da boate Kiss foi classificado pelo Sistema Nacional de Proteção e Defesa Civil como um desastre, tento em vista a decretação de Situação de Emergência pelo município (Brasil, 2013a; Prefeitura de Santa Maria, 2013), com o "[...] objetivo de facilitar as ações de assistência à população afetada pelo evento trágico, com destaque ao auxílio psicológico/psiquiátrico e, indiretamente, financeiro, às famílias vítimas da crise" (Prefeitura de Santa Maria, 2013, art.1).

No presente texto, partindo de um ponto de vista díspar do acima citado e ancorado em definições institucionais nacionais como o Ministério da Integração Nacional (Brasil, 2009; 2012a; 2012b) e órgãos internacionais como a United Nations International Strategy for Disaster Reduction (UNISDR, 2009) e Inter-Agency Standing Committee (IASC, 2007), bem como, considerando a estratificação da magnitude da emergência, trataremos este incêndio pela nomenclatura "evento crítico", ou seja, um "evento que dá início à cadeia de incidentes, resultando no desastre, a menos que o sistema de segurança interfira para evitá-lo ou minimizá-lo" (Brasil, 2009, p. 109).

Eventos críticos de características multifacetadas como esse são, assim, interrupções agudas e abruptas do funcionamento cotidiano de uma sociedade, muitas vezes excedendo a capacidade do coletivo afetado de fazer frente à situação, por meio de seus próprios recursos. Defendemos aqui o ponto de vista de que eles diferem dos desastres ou das catástrofes, que são acontecimentos de maior magnitude e abrangência, em geral atingindo áreas maiores, um grande número de pessoas, ocasionando um elevado número de vítimas fatais e de pessoas afetadas de diversas maneiras, inclusive por provocarem significativa destruição material e desorganização social. Desastres são eventos que, na grande maioria das vezes, necessitam de importante auxílio externo ao território onde ocorreram (UNISDR, 2009). A diferença de nomenclatura se faz importante aqui para embasar a argumentação sobre o tipo de estratégia de resposta no campo psicossocial e saúde mental que iremos descrever.

O termo Saúde Mental (SM), neste artigo, se refere a uma densa e complexa gama de conhecimentos e cuidados que não se limita à psicopatologia ou à semiologia, tampouco ao tratamento dos transtornos 
mentais. Entendemos que esse é um campo de conhecimento e atuação técnica polissêmica, intersetorial e transversal no âmbito das políticas públicas de saúde, abarcando concomitantemente sujeitos e coletividades (Brasil, 2013b; Costa-Rosa, 2000). O termo Atenção Psicossocial (AP), por sua vez, é aqui entendido como tributário de diferentes movimentos sociais e científicos, bem como de distintos campos teóricos, não se restringindo a questões assistenciais, baseando-se no engendramento de vínculos e configurando-se como um campo abrangente de conhecimento e intervenções possíveis, de acordo com as necessidades psicossociais apresentadas por aqueles a quem demandam auxílio neste tema. Neste sentido, utilizamos o termo $\mathrm{AP}$, neste artigo, para abordar o cuidado realizado entre profissionais, usuários e familiares, articulando os processos de vínculo, escuta, negociações de projetos terapêuticos, os embates entre diferentes racionalidades e concepções de ser, de mundo e de sofrimento psíquico, de todos envolvidos (Costa-Rosa, 2000; Grigolo, Delgado, \& Schmidt, 2010).

No que concerne ao método utilizado neste relato de experiência, nos baseamos em paradigmas teórico-metodológicos da abordagem qualitativa, a qual se propõe trabalhar com os significados atribuídos pelos sujeitos aos fatos, relações, práticas e fenômenos sociais, ou seja, preocupa-se com a realidade que não pode ser quantificada, trabalhando com o universo dos significados, motivos, aspirações, valores, atitudes presentes nas relações e ações humanas (Oliveira, 2009). A partir da tradição dos métodos de pesquisa qualitativa usufruímos da observação participante (Whitley, \& Crawford, 2005) e, a partir desta, narramos os acontecimentos como relato de experiência.

\section{Fases da Intervenção}

Como forma de descrição do método utilizado para relatar esta experiência, segmentaremos este relato em três momentos: Pré-evento crítico, no qual são contextualizadas informações pertinentes sobre o local onde aconteceu o evento crítico, na cidade de Santa Maria e sua rede de atenção psicossocial antes do evento; Durante o evento crítico, contextualizando o momento do incêndio até o primeiro mês após; Pós-evento crítico, descrevendo a conjuntura e intervenções nos meses subsequentes ao evento em questão. Na sequência, nos propomos a discutir os resultados oriundos da conformação desta primeira estratégia delineada.

\section{Pré-Evento Crítico: um Panorama de Santa Maria Antes do Incêndio}

Santa Maria, à época, era um município de médio porte da região central do estado do Rio Grande do Sul, com 261.031 habitantes (IBGE, 2010). Entre os diversos dados sociodemográficos que caracterizavam o município, importa destacar o elevado número de profissionais especializados, formados, em sua maioria, em uma das sete instituições de ensino superior presentes no território.

No momento do incêndio, durante o mês de janeiro, período de férias para grande parcela dos trabalhadores brasileiros, o município de Santa Maria passava por um processo de transição de parte da sua equipe gestora de saúde. Além disso, a Rede Municipal de Atenção Básica (RAB) e Rede de Atenção Psicossocial (RAPS) apresentavam consideráveis lacunas, tanto no que concerne à cobertura de serviços quanto em relação ao número de profissionais que compunham as equipes existentes. Soma-se a isso a exiguidade de articulação inter e intrainstitucional quanto ao planejamento, preparação e prontidão das políticas públicas para atuar nas situações de desastres. Como agravante, no momento do evento, a cobertura de Atenção Básica alcançava aproximadamente 123 mil pessoas (48\% da população total), sendo que 52 mil (21\%) encontravam-se sob os cuidados de equipes da Estratégia Saúde da Família - ESF (Brasil, 2016).

A Política Nacional de Atenção Básica (Brasil, 2012c) aponta que a ESF é a forma prioritária de expansão, qualificação e consolidação da Atenção Básica no país. Sua essência está consolidada na formação de uma equipe multiprofissional composta, no mínimo, por um médico, um enfermeiro, um auxiliar de enfermagem e um time de Agentes Comunitários de Saúde (ACS). Sua importância e seu reconhecimento são justificados pela capacidade de ampliação da resolutividade no cuidado, maior impacto positivo na situação de saúde de pessoas e coletividades, melhor relação de custo-efetividade, além de estar alinhada aos princípios do Sistema Único de Saúde (SUS).

Naquele momento, o município também contava com um ambulatório de saúde mental cuja equipe era formada por um grande número de especialistas, trabalhando sem articulação em rede com os demais serviços, sem oferta de dispositivos terapêuticos para além do atendimento individual, e sem um trabalho georreferenciado do território. No que tange ao componente hospitalar da AP e SM, Santa Maria contava 
com 25 leitos habilitados na Casa de Saúde, em processo de adaptação às novas diretrizes do Ministério da Saúde para leitos de SM integrados em hospitais gerais. Havia também outros 25 leitos de saúde mental e uma emergência psiquiátrica 24 h no Hospital Universitário de Santa Maria (HUSM).

Tais fragilidades na composição da rede, do modelo de atenção, da gestão das políticas e de articulação intra e interinstitucional não impediram que os gestores e trabalhadores do SUS se implicassem no processo de construção e implantação de uma estratégia de oferta de cuidado psicossocial e de saúde mental para os sobreviventes e familiares. Tampouco impossibilitou que a estratégia ofertada, que foi executada com grande participação de profissionais voluntários durante os dois primeiros meses que sucederam o evento, tivesse a coordenação de um núcleo de gestão pública tripartite, assessorado, brevemente, a partir da experiência de duas psicólogas representantes uma organização humanitária internacional.

\section{Durante o Evento Crítico: do Momento do Incêndio ao Final do Primeiro Mês}

A primeira reunião da equipe psicossocial junto aos demais membros do Gabinete de Crise aconteceu no amanhecer do dia 28 de janeiro de 2013 e nela estavam presentes membros da gestão municipal, estadual e federal de saúde. A reunião teve como objetivo compartilhar o quadro específico de cada uma das áreas prioritárias para a saúde. Sendo assim, cada um dos gestores informou suas principais demandas e dificuldades e encaminhou suas questões a fim de que o coletivo formado auxiliasse na oferta de soluções rápidas e articuladas. Também foram discutidas diversas estratégias de resposta possíveis para a área da saúde.

No que concerne à AP e SM, foram mapeadas as estruturas e serviços que poderiam ser acionadas como apoio. Esse mapeamento teve como intuito contemplar os primeiros cuidados psicossociais, levando em consideração as reações e demandas previstas na literatura e na experiência empírica das autoras, as quais podem emergir de um evento como esse (IASC, 2007), bem como se considerou a compreensão sobre o modo de funcionamento da Política Nacional de Proteção e Defesa Civil (Brasil, 2012b). A fim de ofertar suporte técnico aos municípios nas demandas de atendimento e cuidado aos sobreviventes e familiares, a Coordenação Estadual de Saúde Mental se prontificou a assumir a coordenação inicial da estratégia de AP e SM. Nesse mesmo dia, diversos profissionais, entidades não governamentais e voluntários se reuniram com o intuito de estruturar uma rede de suporte e cuidados às pessoas afetadas (in)diretamente pelo evento, bem como para aquelas que necessitavam de informações técnicas e profissionais para auxiliar no cuidado de familiares e amigos mobilizados pelo evento. Estavam presentes, entre outros, membros da Diretoria Estadual de Saúde, da Coordenação Estadual de Saúde Mental, membros do Conselho Regional de Psicologia do Rio Grande do Sul, psicólogas(os) voluntárias(os) de diversas organizações da área da saúde e membros da ajuda humanitária internacional. Nessa reunião, foi possível iniciar a elaboração da primeira estratégia de cuidados psicossociais e de saúde mental direcionados aos afetados pelo incêndio na boate.

Naquele momento, foram definidos e pactuados eixos de cuidado e equipes de trabalho para conformação desta rede, os quais deveriam funcionar de acordo com os princípios estruturantes do SUS. Esses eixos, acordados entre todos e descritos a seguir, consolidaram-se da seguinte forma: núcleo de gestão da estratégia de saúde mental; educação permanente; regulação de saúde mental; e seis eixos de apoio psicossocial:

1. Núcleo de Gestão: Constituído por membros da SM e AP dos três entes federados e duas representantes de uma organização internacional (responsáveis pelo primeiro diagnóstico situacional e auxílio na elaboração da estratégia). O trabalho dessa equipe consistiu na definição das ações, na construção de boletins diários de informações para o Gabinete de Crise e no alinhamento dos fluxos de trabalho, além da organização de reuniões duas vezes ao dia (na primeira semana) com os representantes de cada um dos outros eixos, a fim de compreender as demandas e necessidades cotidianas da emergência. Tal grupo foi responsável, também, por auxiliar na produção de indicadores e dados que possibilitassem ao Gabinete de Crise compreender a problemática da demanda psicossocial e dos possíveis encaminhamentos institucionais.

2. Educação Permanente: Este eixo foi organizado de forma transversal, de modo a produzir formações básicas para os profissionais e voluntários que 
estavam atuando na resposta direta ao evento. A primeira formação foi elaborada de forma a contemplar cada eixo da estratégia e, nesses termos, foi estruturada a partir do diagnóstico situacional das estruturas de SM e AP em funcionamento, da escuta das demandas das equipes, da discussão acerca da importância da atuação articulada com demais setores, sempre conforme os princípios do SUS e, por fim, considerando as reações psíquicas esperadas numa situação desta natureza e os cuidados psicossociais a serem ofertados nas primeiras semanas após o evento. Foram organizados, concomitantemente, grupos de supervisão presencial, apoio institucional e matricial (Campos, \&Domitti, 2007), por meio de visitas e da discussão de casos via telefone e internet, bem como videoconferências sobre temas pertinentes aos trabalhadores e voluntários, visando o alinhamento das estratégias de suporte aos afetados, com foco nos sobreviventes e familiares das vítimas.

3. Regulação de Saúde Mental: Uma equipe composta por profissionais contratados e profissionais do município, conhecedores dos trâmites operacionais da Secretaria Municipal de Saúde, foi responsável por encaminhar e auxiliar no fluxo de referência das pessoas que buscavam informações e cuidados de AP e SM por telefone e/ou presencialmente em qualquer uma das estruturas físicas da rede de cuidados emergenciais. Este eixo funcionou a partir de princípios similares aos da regulação em saúde pública, ou seja, entendendo por "regulação" a equipe responsável pela organização da oferta de cuidados e fluxos, buscando garantir o acesso dos usuários aos serviços de saúde de acordo com as necessidades singulares.

4. Apoio Psicossocial: Este eixo foi dividido em seis subeixos:

4.1. Nos territórios dos ritos de despedida disponibilizou-se suporte psicossocial aos familiares e amigos das vítimas no decurso dos velórios, missas e atos públicos. Por entendermos que um evento dessa natureza pode provocar reações agudas de sofrimento, optou-se por enviar, a cada um destes rituais, profissionais que se mantinham presentes sem ofertar assistência de forma ostensiva, mas que pudessem acolher as manifestações de sofrimento dos presentes que desejassem apoio e auxiliá-los a compreender aquele processo como uma reação esperada à dor da perda. Em casos específicos, esses profissionais também poderiam encaminhar as pessoas, que assim o solicitassem, a espaços de cuidado em saúde onde poderiam ser acompanhadas por uma equipe qualificada.

4.2. Nas UPAS e SAMU, foi nomeado um grupo de psicólogos e outros profissionais de saúde voluntários que ficaram responsáveis por prestarem apoio qualificado aos profissionais das Unidades de Pronto Atendimento (UPA) e Serviço de Atendimento Móvel de Urgência (SAMU), aos afetados, familiares, amigos e outras pessoas demandantes de apoio nesses locais. Ressalta-se que uma parte considerável desses atendimentos consiste em casos nos quais a população ou os profissionais identificavam no corpo sinais e sintomas que, por vezes, eram expressões de sofrimentos emocionais, como por exemplo, crises de insônia, problemas gastro-intestinais, dores no corpo não diagnosticáveis pela clínica médica, taquicardia, hipervigilância, agitação etc.

4.3. Nos hospitais organizou-se também uma equipe de psicólogas(os) voluntárias (os) com o objetivo de prover um suporte aos cuidadores e familiares dos pacientes internados, tendo em vista que a maior parte desses pacientes encontrava-se em estado grave. Parte dessa equipe também ficou disponível para o suporte aos profissionais de saúde que manifestassem demandas de apoio matricial relacionadas a esse tipo de atendimento e/ou aos pacientes internados referenciados pela equipe médica.

4.4. Serviço de acolhimento 24 horas (Plantão de Saúde Mental), serviço criado em decorrência das fragilidades e lacunas da RAPS no município, mencionadas anteriormente, o qual funcionou como um serviço complementar aberto 24 horas, sete dias por semana. Este serviço foi desenhado para acolher e atender especificamente os casos que apresentavam queixas de sofrimento agudo relacionado ao evento, por meio de uma equipe multiprofissional, inicialmente formada por alguns profissionais do município - que foram realocados de seus servi- 
ços de saúde pública - e por profissionais voluntários. Em um primeiro momento, foi sugerido por gestores municipais alocar esse serviço em uma sala dentro do Centro Desportivo Municipal, isto é, no mesmo local onde foram identificados e velados os corpos das vítimas. Por esse motivo, optou-se por fazê-lo em outro espaço, menos relacionado a cenas de intenso sofrimento.

O espaço escolhido foi o segundo pavimento do edifício do Centro de Atenção Psicossocial (CAPS) "Caminhos do Sol", que havia sido alugado pela Prefeitura de Santa Maria anteriormente. Para esse serviço delineado, em primeira instância, para ser executado apenas por psicólogas(os) voluntárias(os), elaborou-se uma escala com uma equipe de psicólogas(os), assistentes sociais, enfermeiras(os), técnicas(os) de enfermagem e médicas(os) voluntárias(os) em sistema de plantão, cobrindo o período de 24 horas por dia. Tal estrutura localizava-se a apenas 1,6 quilômetro do Centro Desportivo Municipal, facilitando o deslocamento daqueles que estavam e/ou haviam estado presentes durante os velórios e reconhecimentos coletivos de corpos.

Fazia parte do escopo de ofertas de cuidado dessa equipe, além das escutas individuais, familiares ou em grupo, o acolhimento de AP e SM por telefone e visitas domiciliares. $\mathrm{O}$ acolhimento via telefone foi estabelecido a fim de garantir a prontidão de uma primeira escuta e o encaminhamento adequado quando necessário. Já nas visitas domiciliares atendiam-se demandas expressas via telefone, ou identificadas nos atendimentos efetuados no local, com a finalidade de acolher e capacitar as famílias para utilizarem seus próprios recursos, bem como informar sobre a possibilidade de um atendimento especializado. Assim, além de abrigar as equipes de visita domiciliar, esse espaço alojou também a regulação do serviço de encaminhamento para as internações hospitalares dos casos de SM.

Como afirmado anteriormente, havia no município 50 leitos psiquiátricos, sendo que 25 não estavam apropriados para o uso e, além desses leitos já disponibilizados pela rede, no momento do incêndio, disponibilizou-se o acesso a dois leitos psiquiátricos no Hospital São Francisco de Assis. Ressalta-se que, nos três primeiros meses após o evento, apenas seis casos foram referenciados para internação em leitos psiquiátricos sendo que todos esses usuários já pos- suíam importante histórico de sofrimento psíquico anterior à data do evento (Santa Maria, 2013).

4.5. Foco na Atenção Básica, foi realizado com a configuração de uma equipe exclusiva para sensibilização de profissionais da atenção básica oferecendo apoio a atendimentos clínicos, quando necessário, e priorizando os cuidados a médio $\mathrm{e}$ longo prazo, a nível municipal e regional. Uma vez que nem todas as vítimas do incêndio ou seus familiares residiam no município de Santa Maria, a abrangência das ações passou do âmbito municipal para o regional.

$\mathrm{Na}$ primeira semana após o incêndio, estabeleceu-se contato com equipes da rede SUS e elegeram-se, entre voluntários e profissionais da atenção básica, coordenadores de equipes, os quais, junto com Agentes Comunitários de Saúde, Enfermeiros e Técnicos de Enfermagem, somaram-se a um grupo de psicólogas(os) voluntárias(os) e equipe da assistência social para prover atendimentos e cuidados.

Nas semanas que se seguiram, especificamente em Santa Maria, formaram-se cinco equipes com profissionais voluntários e servidores do município cuja função principal foi delinear um mapeamento das famílias locais que haviam perdido entes queridos no EC. Essa equipe encontrou 62 casos apenas na cidade de Santa Maria. Além disso, em parceria com a Universidade Federal de Santa Maria, foi possível integrar o apoio de profissionais da instituição, e disponibilizar um veículo, o que facilitou o deslocamento das equipes para visitas domiciliares e de matriciamento, objetivando assegurar a retaguarda especializada a equipes e profissionais encarregados da atenção a problemas de saúde nas Unidades Básics de Saúde (UBS) e nas ESF (Campos, \& Domitti, 2007).

4.6. Apoio às equipes de resposta ao evento, considerando que a maior parte dos profissionais e voluntários presentes nas estratégias de resposta ao incêndio nunca havia trabalhado em situações semelhantes, estruturou-se um grupo responsável por promover ações de sensibilização e informação específica para essas pessoas, acerca do cuidado em situação de crise. Esse grupo criou também estratégias de acolhimento às equipes (bombeiros, policiais, motoristas, maqueiros, equipes de saúde...) envolvidas nos atendimentos aos afetados. 
A metodologia da estratégia descrita nos quatro eixos acima, na prática, funcionou durante os dois primeiros meses após o incêndio, sob a articulação do Núcleo de Gestão, acima citado e, aos poucos, foi sendo adaptada às mudanças do contexto local.

\section{Pós-Evento Crítico: Acompanhamento e Reestruturação}

Segundo mês após o evento: Este momento foi caracterizado pelo início da transição entre a estratégia inicial e uma estratégia mais duradoura. A fim de produzir um cuidado coeso e articulado, a Coordenação Estadual de Saúde Mental, juntamente com as Coordenadorias Regionais de Saúde, assumiu a frente da elaboração, assessoria e distribuição de informações específicas do cuidado psicossocial a todos os municípios afetados. Como primeiro passo, foram levantados dados que possibilitassem mapear o número de municípios com casos registrados de vítimas e sobreviventes e, na sequência, iniciou-se a elaboração de diretrizes técnicas para acompanhamento psicossocial às vítimas e aos familiares do EC e gestão de casos específicos.

Concomitante a isso, as equipes locais iniciaram um processo de avaliação da estratégia e revisão de prontuários de todos os pacientes atendidos durante $o$ primeiro mês após o incêndio, a fim de compreenderem quais foram as principais reações e dificuldades encontradas em um primeiro momento, bem como entenderem se a rotatividade dos voluntários, que caracterizara as ações daqueles primeiros dias, havia possibilitado um acompanhamento coerente com a demanda apresentada pelos usuários daqueles serviços. A proposta também se prestou a mapear os usuários que necessitavam ser recontatados por meio de uma busca ativa, a fim de dar sequência aos processos em consonância com as demandas singulares de cada um.

Destaca-se que independentemente dos ajustes necessários a posteriori, os procedimentos criados estabeleceram a rotatividade dos voluntários como critério pré-definido de atendimento, visto a importância de vincular os usuários ao serviço de acolhimento do SUS (ACOLHE), e não com os psicólogos voluntários, procedimento este definido pela equipe de gestão e entendido como uma estratégia coerente para a demanda, uma vez que o serviço de voluntariado deve limitar-se ao tempo de estruturação do Município, para atendimento dos casos.
Baseando-se na análise dos prontuários foi possível perceber a curva descendente do número de pessoas que buscaram atendimento espontaneamente no serviço $24 \mathrm{~h}$ no fim deste segundo mês, se comparado ao volume de atendimentos do primeiro mês. Em março de 2013, o número de acolhimentos no serviço diminuiu para menos da metade do mês anterior - foram 701 atendimentos em fevereiro e 297 em março. O mesmo se deu com os acolhimentos por telefone-613 em fevereiro e 345 em março - e com as visitas domiciliares - 102 em fevereiro e 43 em março. Quanto às internações de saúde mental, houve apenas cinco encaminhamentos no primeiro mês e um no segundo mês que sucedeu o evento. Por conta disso, o mapa da rede ampliada de AP e de SM foi revisto, e uma busca ativa dos sobreviventes referenciados e/ou autorreferenciados por meio de prontuários hospitalares foi feita, além do que, outras redes de solidariedade comunitárias tornaram-se conhecidas da equipe de gestão da estratégia. Esse período foi ainda caracterizado pelo volumoso número de visitas externas (grupos de estudo, consultores ministeriais, pesquisadores, especialistas...) de distintos setores, o que gerou novos desafios para as equipes de gestão municipal e estadual.

Outro ponto importante dessa etapa de consolidação da estratégia psicossocial foi a abertura de um edital para contratação municipal de profissionais de saúde, tais como: psicólogos, enfermeiros, técnicos de enfermagem, assistentes sociais, médico clínico geral e médico psiquiatra temporários, de forma a garantir a sustentabilidade da ação emergencial que, anteriormente, estava baseada sobretudo na participação de voluntários, mesmo que com uma articulação direta com a política pública municipal de saúde mental (PMSM, 2014). Assim, a partir do que foi acordado no território, a parceria entre os três entes federados deu-se de forma complementar, estendendo-se ainda à educação permanente daqueles recém-contratados.

Terceiro mês após o evento: Nesta etapa da estratégia ocorreu a substituição oficial da equipe de voluntários por meio da contratação municipal de 14 psicólogos, quatro médicos, cinco enfermeiros, três técnicos de enfermagem e dois assistentes sociais para compor a equipe permanente do serviço de acolhimento $24 \mathrm{~h}$, além da transferência desses para outro espaço, de uso exclusivo do Serviço de Acolhimento. Tal mudança exigiu da nova equipe, juntamente com a equipe gestora, a construção da identidade do sexto serviço de SM e AP do município. 
Vale ressaltar que parte dos novos profissionais contratados não acompanhou o processo de resposta desde o início, o que tornou necessário um nivelamento no conhecimento da linha histórica atrelado a uma equiparação das ações, interesses e expertises daqueles que passaram a integrar o serviço. Foi necessário também alinhar o conhecimento acerca da RAPS municipal a fim de organizar um serviço integral e integrado para os usuários.

Durante esse período aumentaram os acolhimentos realizados por telefone (de 345 em março para 446 em abril). Tal processo foi entendido por nós como um efeito da revisão dos prontuários ativos e inativos dos serviços pela equipe contratada como forma de garantir uma linha de cuidado aos sobreviventes e familiares acompanhados. As visitas domiciliares diminuíram de 43 em março para 30 em abril, assim como os atendimentos no serviço (de $297 \mathrm{em}$ março para 289 em abril). Nesse período não houve registro de encaminhamentos para internação de saúde mental.

\section{Discussões e Reflexões acerca dos Resultados dessa Experiência}

Uma intervenção em um EC desencadeia complexos desafios para os profissionais responsáveis pela resposta. Neste sentido, pretendeu-se reforçar a necessidade e a importância de responder a este evento de forma articulada e baseada, sobretudo nas demandas e oferecimentos das equipes locais, mesmo que, como descrito no artigo, houvesse lacunas e dificuldades crônicas no sistema de saúde municipal - o que não difere da situação da maioria dos municípios brasileiros.

Assim, verificou-se ser de fundamental importância que estratégias de resposta a um evento como esse esteja articulada com mecanismos de gestão pública municipal, estadual e federal, a fim de garantir a sustentabilidade e integralidade do processo de cuidado. Isso significa que a ação de qualquer profissional, entre eles a(o) psicóloga(o), independentemente de onde ela parta (sociedade civil, organismos nacionais e/ou internacionais), precisa estar estruturalmente coordenada e inserida em uma proposta de cuidado que seja orgânica ao modelo nacional, integrando o amplo guarda-chuva das políticas públicas brasileiras (Weintraub, Noal, Vicente, \& Knobloch, 2015). Entendemos ser esse provavelmente o principal desafio de uma intervenção nessas condições, mas é também a ação mais importante para torná-la profícua e relevante para a população em questão.

Santa Maria, por sua vez, recebeu profissionais de saúde voluntários, oriundos de vários estados do Brasil, além de outros profissionais que se apresentavam como pesquisadores e especialistas na área de desastres desde as primeiras horas pós o incêndio. Muitos desses profissionais se inseriram na estratégia tripartite organizada pelo poder público para garantir $a$ atenção às demandas psicossociais desencadeadas pelo EC. Contudo, como é bastante comum em situações dessa natureza (Molina, 2011; Noal et al., 2016), muitos profissionais e pesquisadores empreenderam ações e estratégias isoladas e de forma paralela, desvinculadas das ofertas e dos fluxos firmados pela equipe gestora central, acarretando em ações sobrepostas e intervenções despreocupadas com uma atenção integral e integrada à população atendida. Inúmeras iniciativas, desvinculadas da estratégia central, colocaram seu acento na abordagem de pressupostos psicopatológicos decorrentes do evento, focando suas ações na oferta de atendimento individual e/ou vitimizador, desconsiderando muitas vezes a capacidade de resposta das estratégias coletivas, das redes socioafetivas e/ou comunitárias (Udomratn, 2008).

Reforça-se que as propostas de intervenção nesse campo devem ter como um de seus pilares fundamentais a construção de alternativas para auxiliar na elaboração dos sofrimentos gerados pela situação, sejam eles coletivos ou individuais. Da mesma forma, é preciso que essas propostas sejam compatíveis com a demanda existente, na direção da autonomia das comunidades, grupos de pessoas e autoridades envolvidos frente ao que aconteceu (Reyes, 2006a; 2006b). Avaliamos que, enquanto ator social dessa composição de cuidado, o profissional da AP tem como ações basilares: escutar as demandas, considerar a existência do desespero, da tristeza, da dor e do luto, conhecer estratégias locais de cuidado, mapear a rede de possibilidades ampliadas de atenção e articular de forma sustentável suas ações. Recomenda-se que toda ação de resposta a uma emergência não deve perder de vista a recuperação e a reconstrução do cenário e do contexto onde o evento ocorreu (IASC, 2007; Brasil, 2012b).

Especificamente na intervenção em Santa Maria, percebe-se que, no período anterior ao evento, nenhum dos três entes federados dispunha de um plano práticoloperacional psicossocial elou um guia de diretrizes e condutas em saúde mental para emergências e 
Noal, D.S.; Vicente, L.N.; Weintraub, A.C.A.M.; Fagundes, S.M.S.; Cabral, K.V.; Simoni, A.C.R.; Pacheco, M.L.L.; Pedroza, R.L.S.; Pulino, L.H.C.Z. (2016). Atenção psicossocial afetados Kiss.

desastres. Entretanto, embora não houvesse um plano a priori, as atividades e ações elaboradas como resposta foram estruturadas de forma condizente com as necessidades psicossociais do público-alvo, bem como em consonância com as diretrizes (inter) nacionais (IASC, 2007) e a legislação brasileira (Brasil, 2012; 2013), com enfoque na criação de uma estratégia que pudesse ser sustentável em médio prazo e efetivamente beneficiasse a população atendida.

Um ponto relevante que pode ser entendido como consequência dessa estratégia foi o fortalecimento da estruturação da rede de SM e AP (Schneider, 2009). A célere execução da estratégia que propunha uma ampla capilaridade do cuidado psicossocial, e que deu foco às estruturas locais, foi mais um dos resultados considerados, pelas autoras, como extremamente positivo e eficiente na gestão do cuidado coletivo e singular, visto que, entre outras especificidades, deixou-se evidente à população a oferta disponível e ao mesmo tempo propôs-se a acompanhar de formas distintas as demandas emergentes às diferentes etapas e características do cuidar em eventos e emergências complexas.

Ainda sobre os destaques dessa estratégia, a revisão de prontuários elaborados durante a fase aguda por voluntários e reavaliados pela equipe contratada, com o objetivo de criar estratégias de continuidade do cuidado, foi outro resultado relevante. Com isso, além de avaliar a gravidade dos casos e os pontos nevrálgicos do cuidado inicial, foi possível acompanhar a continuidade dos atendimentos realizados por voluntários, bem como as possíveis rupturas e encaminhamentos ofertados pelos distintos psicólogos que atenderam aqueles usuários. Desse modo, a busca ativa foi importante para o restabelecimento do processo de cuidado, por vezes fraturado pela descontinuidade da presença do cuidador inicial.

Avaliamos ainda que a implantação dos serviços de plantão 24 horas foi um dos resultados primordiais do desenho da estratégia no momento inicial da resposta, atuando de forma eficaz e precisa, durante o primeiro acolhimento e encaminhamento adequado quando necessário. Como seu principal foco foi, ao mesmo tempo, apoiar os cidadãos na utilização de seus recursos próprios e comunitários e também informá-los da possibilidade de um atendimento especializado caso necessário, entendemos que isso fomentou a autonomia e a resiliência singular de cada usuário.

Ainda assim, em nossa avaliação, algumas dificuldades foram encontradas na implantação da estratégia como um todo. Um primeiro desafio foi a presença de um grande número de atores externos como: consultores, pesquisadores, apoiadores, voluntários, os quais se apresentaram, pelas mais diversas vias, e em diferentes fases do evento, propondo-se a auxiliar na resposta, no entanto, despreocupados em fazer parte de um sistema integral. Vale comentar que cada um destes grupos se interessava, na maioria das vezes, apenas por uma oferta específica e pré-definida a uma avaliação do evento em questão, não denotando nenhuma correlação com a estratégia já definida e sem preocupação em conhecer as ações já realizadas ou existentes. Isso, muitas vezes, acarretava em sobreposição de ações ou ações contraproducentes para os afetados.

Observamos que, quando esses atores externos pautam sua intervenção a partir de perspectivas próprias e não a partir da demanda da situação vigente, possivelmente serão geradas contradições e fragmentações da oferta de serviços, bem como uma dificuldade inicial de construção de uma identidade própria à demanda local e, quiçá, sensação de insegurança por parte dos afetados e dos profissionais locais, visto que as ofertas não condizem com suas necessidades. Somou-se a isso a alta rotatividade de profissionais locais, algo bastante recorrente em respostas a emergências (Goyet, 2000).

Outro ponto a ser analisado versa sobre a rotatividade de gestores na Coordenação de Saúde Mental, o que se tornou um desafio maior ainda para a conformação de uma estratégia a médio e longo prazo. O acompanhamento e a identificação de responsáveis por atividades, ações e políticas de voluntários e trabalhadores da RAPS são dificultados quando não há um elemento fixo na gestão do início ao término do processo, especialmente nas primeiras quatro semanas após um evento dessa magnitude, acarretando ruídos de comunicação e dificultando parte das atividades de acompanhamento (Goyet, 2000). Por isso é preciso estar preparado para lidar com essa rotatividade antes, durante e depois dos eventos desta natureza.

$\mathrm{O}$ fato de ter havido em Santa Maria um trabalho intensivo de cogestão tripartite de uma rede frágil, convocada a se reorganizar urgentemente para dar conta das necessidades da população atingida, a nosso ver, contribuiu para fortalecer a rede municipal, produzindo efeitos que ainda estão presentes, passado três anos do início do evento, nos serviços de saúde. Cabe ressaltar que não foi objetivo deste relato avaliar o impacto clínico da estratégia para a população-alvo, mas sim refletir sobre as estratégias postas em prática nos primeiros três meses 
após o evento crítico. Tal avaliação dos resultados dessa estratégia para os indivíduos que por ela foram atendidos seria bastante interessante visto que, na maioria das vezes, não é fácil encontrar esse tipo de informação na literatura (Favero, Sarriera, \& Trindade, 2014).

Reconhecemos, no entanto, o tímido, porém crescente, número de publicações como: manuais e protocolos de trabalho para profissionais da AP e SM em situações de emergência (IASC, 2007; OMS, 2011; WHO, WTF \& WVI, 2011; 2013; WHO \& UNHCR, 2012; 2015), bem como a ascendência no número de debates sobre as diferentes perspectivas teórico-práticas para a atuação desses profissionais (Jones, Asare, El Masri \& Mohanraj, 2007; Jones et al., 2009). Os manuais e protocolos abordam problemas da atuação da AP e SM em emergências, como a fragmentação, a falta de conhecimento técnico das equipes e a falta de coordenação com políticas públicas locais, entretanto, o debate, sobretudo na literatura internacional, parece girar em torno de dois eixos: quais são as principais correntes teórico-práticas que demonstram efetividade nesse campo e, mais recentemente, como atender o sofrimento da emergência sem esquecer dos usuários anteriores. O relato apresentado aqui perpassa esses pontos centrais e, na sequência, aponta recomendações a partir da análise das autoras.

\section{Algumas Conclusões e Recomendações}

Como conclusões finais pontuamos que intervenções, estratégias e ações, elaboradas como estratégia de resposta ao evento crítico do incêndio na Boate Kiss foram estruturadas de forma condizente com as necessidades da população afetada, bem como foram coerentes com as diretrizes e políticas (inter) nacionais, uma vez que foram propostas levando em consideração o enfoque na criação de uma estratégia articulada e sustentável em um curto e médio prazo, visando o bem-estar psicossocial e de saúde mental da população cuidada.

Consideramos ainda que, apesar das fragilidades crônicas na composição da rede de saúde mental e atenção psicossocial, os gestores, trabalhadores do SUS e voluntários conseguiram implantar uma estratégia de oferta de cuidado condizente com as demandas emergenciais da população afetada.

Nesses termos, a partir dessa experiência, e de outras (Weintraub et al., 2015), sugerimos a seguir alguns pontos que consideramos relevantes para a criação, implementação e produção de estudos e pesquisas que auxiliem na conformação de estraté- gias condizentes com as respostas a eventos críticos e desastres no Brasil. São eles:

- Enfoque geral e interinstitucional na promoção de AP e Saúde Mental Coletiva (Fagundes, 2010), a fim de conformar um processo construtor de sujeitos sociais desencadeadores de transformações nos modos de pensar, sentir e fazer política, ciência e gestão no cotidiano das estruturas de mediação da sociedade, extinguindo e substituindo as práticas tradicionais por outras capazes de contribuir para a criação de projetos de vida.

- Estudos e intervenções que levem em consideração a percepção de cuidado voltado para as demandas dos usuários, retirando o foco simplista no sofrimento e na patologização das reações esperadas num pós-evento crítico e voltando-se para as reações esperadas, potencialidades e resiliências da população afetada.

- Articulação e parceria entre os três entes federados na elaboração preventiva de planos de contingência, como é previsto pela legislação nacional de Proteção e Defesa Civil (Brasil, 2012b), contemplando, inclusive, ações de SM. A existência de um plano irá, sem dúvida, contribuir para uma oferta de serviços de apoio de qualidade e com maior rapidez na eventualidade de qualquer tipo de desastre ou evento menor que possa acometer o município.

- Proposição de estratégias de resposta elaboradas de forma a contemplar as demandas emergentes a médio ou mesmo longo prazo, e não apenas voltadas aos primeiros momentos pós-evento. Acreditamos ainda que um estudo das portas de entrada dos usuários, isto é, entender quais são os locais dentro da estrutura da rede SUS entendidos pela população afetada como lugar de cuidado psicossocial, pode facilitar a oferta dos serviços dessa natureza durante um evento crítico, posto que seria possível garantir educação permanente voltada para o tema desses profissionais, aumentando a capacidade da produção de cuidado específico para essas situações.

- No momento da resposta, formação de uma gestão da crise articulada com voluntários, profissionais da rede SUS e outros apoiadores que venham a contribuir com a efetivação da estratégia. 
Noal, D.S.; Vicente, L.N.; Weintraub, A.C.A.M.; Fagundes, S.M.S.; Cabral, K.V.; Simoni, A.C.R.; Pacheco, M.L.L.; Pedroza, R.L.S.; Pulino, L.H.C.Z. (2016). Atenção psicossocial afetados Kiss.

Sugere-se, ainda, realizar grupos de trabalho com outros atores da gestão municipal a fim de melhorar a prevenção, mitigação, preparação, resposta e reconstrução de territórios sujeitos a eventos críticos. Além do Sistema de Proteção e Defesa Civil, citado acima, a Assistência Social, a Educação, a Habitação, a Cultura, entre outras secretarias, podem contribuir consideravelmente para a estruturação tanto de um plano de prevenção quanto da preparação para possíveis eventos dessa natureza. Espera-se, assim, que os campos da AP e da SM possam contribuir com a diminuição dos efeitos nocivos para a saúde que um evento como esse pode gerar e, sobretudo, contribuir para a efe-

\section{Referências}

Brasil. (2012a). Instrução Normativa $n^{\circ} 1$, de 24 de agosto de 2012. Estabelece procedimentos e critérios para a decretação de situação de emergência ou estado de calamidade pública pelos Municípios, Estados e pelo Distrito Federal, e para o reconhecimento federal das situações de anormalidade decretadas pelos entes federativos e dá outras providências. Diário Oficial da União, 30 ago..

Brasil. (2012b). Lei no 12.608, de 10 de abril de 2012. Institui a Política Nacional de Proteção e Defesa Civil - PNPDEC; dispõe sobre o Sistema Nacional de Proteção e Defesa Civil - SINPDEC e o Conselho Nacional de Proteção e Defesa Civil - CONPDEC; autoriza a criação de sistema de informações e monitoramento de desastres; altera as Leis $\mathrm{n}^{\mathrm{o}}$ 12.340, de $1^{\circ}$ de dezembro de 2010, 10.257, de 10 de julho de 2001, 6.766, de 19 de dezembro de 1979, 8.239, de 4 de outubro de 1991, e 9.394, de 20 de dezembro de 1996; e dá outras providências. Brasília: Diário Oficial da União, 11 abr.

Brasil. (2013a). Portaria no 21, de 4 de fevereiro de 2013. Reconhece Situação de Emergência no município de Santa Maria - RS. Diário Oficial da União, 05 fev.

Brasil. Ministério da Integração Nacional. (2009). Secretaria Nacional de Defesa Civil. Glossário de defesa civil estudos de riscos e medicina de desastres. Brasília, DF: Autor.

Brasil. Ministério da Saúde. (2012c). Política Nacional de Atenção Básica (Série E. Legislação em saúde). Brasília, DF: o autor. tiva garantia de direitos das pessoas em situação de risco de desastres.

Avaliamos que a experiência da criação, implantação e continuidade da estratégia de atenção psicossocial elaborada em Santa Maria, como resposta ao incêndio da boate Kiss em 2013, possa servir de dispositivo analisador das demandas e possibilidades que um evento dessa magnitude oferece, vindo ao auxílio da conformação de novas estratégias de cuidado em EC. Esperamos, assim, que esse relato de experiência possa contribuir com a formação e reflexão de outros profissionais locais, gestores do SUS, voluntários e trabalhadores de organizações não governamentais que tenham interesse ou que venham a trabalhar nessas situações.

Brasil. Ministério da Saúde. (2013b). Secretaria de Atenção à Saúde. Departamento de Atenção Básica. Saúde mental (Cadernos de atenção básica, 34). Brasília, DF: o autor.

Brasil. Portal da Saúde (2016). Departamento de Atenção Básica. Histórico de cobertura da saúde da família. Recuperado de: http://dab.saude.gov.br/portaldab/historico_cobertura_sf.php

Campos, G.W.S., \& Domitti, A. C. (2007). Apoio matricial e equipe de referência: uma metodologia para gestão do trabalho interdisciplinar em saúde. Cadernos de Saúde Pública, 23(2), 399-407. doi:10.1590/S0102-311X2007000200016

Costa-Rosa, A. (2000). O modo psicossocial: um paradigma das práticas substitutivas ao modo asilar. In $\mathrm{P}$. Amarante (Org.), Ensaios: subjetividade, saúde mental, sociedade. Rio de Janeiro, RJ: Fiocruz.

Fagundes, S. (2010). Os municípios e a desinstitucionalização em saúde mental coletiva. In F. Campos, \&A. Lancetti (Orgs), Saúde e loucura: experiências da reforma psiquiátrica (pp. 203-231). São Paulo, SP: Hucitec.

Favero, E., Sarriera, J. C., \& Trindade, M. C. (2014). O desastre na perspectiva sociológica e psicológica. Psicologia em Estudo, 19(2), 201-209. doi:10.1590/1413-737221560003

Goyet,C.V. (2000).Stop propagatingdisastermyths. Lancet, 356(9231),762-764.doi:10.1016/S0140-6736(00)02642-8

Grigolo, T., Delgado, P. G., \& Schmidt, M. (2010). Avaliar CAPS: um retrato do funcionamento da rede dos serviços substitutivos no Brasil. In F. Campos, \& A. Lancetti (Orgs.), Saúde e loucura: experiências da reforma psiquiátrica (pp. 371-390). São Paulo, SP: Hucitec. 
Instituto Brasileiro de Geografia e Estatística - IBGE. (2010). Sinopse do censo demográfico 2010. Rio de Janeiro, RJ: o autor. Recuperado de http://censo2010.ibge.gov.br/

Inter-Agency Standing Committee - IASC. (2007). IASC Guidelines on mental health and psychosocial support in emergency settings. Geneva: WHO.

Jones,L.,Asare,J.,Elmasri, M.,\& Mohanraj,A. (2007).Mental health in disaster settings. British Medical Journal, 335(7622), 679-680. doi:10.1136/bmj.39329.580891.BE

Jones, L., Asare, J. B., Elmasri, M., Mohanraj, A., Sherief, H. \& Van Ommeren, M. (2009). Severe mental disorders in complex emergencies. Lancet, 374(9690), 654-61. doi:10.1016/S0140-6736(09)61253-8

Molina, R. (2011). A psicologia das emergências e desastres e compromisso social: a experiência latino-americana In Conselho Federal de Psicologia, Psicologia das emergências e desastres na América latina: promoção de direitos e construção de estratégias de atuação. Brasília, DF: o autor.

Noal, D.S., Vicente, L. N., \&Weintraub, A. C. M. (2016). Ajuda que vem de "fora": a conformação da primeira estratégia psicossocial e de saúde mental pós-incêndio na Boate Kiss. In G. Mafacioli, M. F. Lüdtke, M. L. L. Pacheco, M. M. Sanfelice, \& V. A. Dassoler (Orgs.), A integração do cuidado diante do incêndio na Boate Kiss: testemunhos e reflexões (p. 314). Curitiba: CRV.

Oliveira, M. H. P. (2009). A representação de si mesmo em narrativas autobiográficas de escritores brasileiros. Memorandum, 17, 107-121.

Organização Mundial daSaúde-OMS. (2011). Psychosocial first aid: guide for field workers. Geneva: $\mathrm{o}$ autor.

Paranhos, M. E., \& Werlang, B. S. G. (2015). Psicologia nas emergências: uma nova prática a ser discutida. Psicologia: Ciência e Profissão, 35(2), 557-571. doi:10.1590/1982-370301202012

Prefeitura de Santa Maria. (2013). Decreto Executivo $n^{\circ} 10$, de 1 de fevereiro de 2013. Decreta Situação de Emergência no Município afetado por incêndios urbanos (COBRADE - 2.3.1). Diário Oficial do Município de Santa Maria, 2 fev.

Reyes, G. (2006b). Conclusions and recommendations for further progress. In G. Reyes, \& G. A. Jacobs (Eds.), Handbook of international disaster psychology: interventions with special needs populations (pp. 141-149). Westport, CT: Praeger.
Reyes, G. (2006a). Foreword. In G. Reyes, \& G. A. Jacobs (Eds.), Handbook of international disaster psychology: fundamentals and overview (pp. 13-14). Westport, CT: Praeger.

Schneider,A.R. (2009) Arede de atenção em saúdemental: a importância da interação entre a atenção primária e os serviços de saúde mental. Ciência e Saúde, 2(2):78-84. doi:10.15448/1983-652X.2009.2.4843

Santa Maria. Secretaria Municipal. (2013). Relatório do Serviço Acolhe Saúde. Santa Maria, RS: o autor.

Tol, W.A., Barbui, C., Galappatti, A., Silove, D., Betancourt, T. S., Souza, R. et al. (2011). Mental health and psychosocial support in humanitarian settings: linking practice and research. Lancet, 378(9802), 1582-91. doi:10.1016/S0140-6736(11)61094-5

Udomratn, P. (2008). Mental health and the psychosocial consequences of natural disasters in Asia. International Review of Psychiatry, 20(5): 441-444. doi:10.1080/09540260802397487

United Nations International Strategy for Disaster Reduction-UNISDR. (2009). Terminología sobre reducción del riesgo de desastres. Recuperado de http://www.unisdr. org/files/7817_UNISDRTerminologySpanish.pdf

Van Ommeren, M., Saxena, S., \& Saraceno, B. (2005). Aid after disasters: needs a long term public mental health perspective. Br Med J, 330(7501), 1160-1161. doi:10.1136/bmj.330.7501.1160

Weintraub, A.C.M., Noal,D. S.,Vicente, L. N., \&Knobloch, F. (2015). Atuação do psicólogo em situações de desastre: reflexões a partir da práxis. Interface (Botucatu), 19(53), 278-298 . doi:10.1590/1807-57622014.0564

Whitley, R. \& Crawford, M. (2005). Qualitative research in psychiatry. Canadian Journal of Psychiatry, 50(2), 108-14. doi:10.1177/070674370505000206

World Health Organization V WHO, War Trauma Foundation-WTF,\&WorldVision International-WVI (2013). Psychological first aid: facilitator's manual for orienting field workers. Geneva: o autor.

World Health Organization V WHO, War Trauma Foundation-WTF, \&WorldVision International-WVI (2011). Psychological first aid: guide for field workers. Geneva: o autor.

World Health Organization - WHO, \& United Nations High Commissioner for Refugees - UNHCR. (2012). Assessing mental health and psychosocial needs and resources: toolkit for major humanitarian settings. Geneva: o autor. 
Noal, D.S.; Vicente, L.N.; Weintraub, A.C.A.M.; Fagundes, S.M.S.; Cabral, K.V.; Simoni, A.C.R.; Pacheco, M.L.L.; Pedroza, R.L.S.; Pulino, L.H.C.Z. (2016). Atenção psicossocial afetados Kiss.

World Health Organization - WHO, \& United Nations High Commissioner for Refugees - UNHCR. (2015). mhGAP Humanitarian Intervention Guide (mhGAP-HIG): clinical management of mental, neurological and substance use conditions in humanitarian emergencies. Geneva: o autor.

\section{Débora da Silva Noal \\ Doutoranda em Processos do Desenvolvimento Humano e Saúde pela Universidade de Brasília, Brasília - DF. Brasil. \\ E-mail: noaldebora@gmail.com \\ Letícia Nolasco Vicente \\ Especialista pelo instituto Sedes Sapientiae, São Paulo - SP. Brasil. \\ E-mail: nolasco.leticia@gmail.com}

\section{Ana Cecília Andrade de Moraes Weintraub}

Doutoranda em Psicologia Escolar e do Desenvolvimento Humano, Universidade de São Paulo, São Paulo - SP. Brasil.

E-mail: ana.cecilia.moraes@usp.br

\section{Sandra Maria Sales Fagundes}

Mestre em Educação pela Universidade Federal do Rio Grande do Sul, Porto Alegre - RS. Brasil.

E-mail: sandrafagundes@cpovo.net

\section{Károl Veiga Cabral}

Doutora em Antropologia Médica eSaúde Internacional pela Universitat Rovira i Virgili. Espanha.

E-mail: karolveigacabral@gmail.com

\section{Ana Carolina Rios Simoni}

Doutora em Educação pela Universidade Federal do Rio Grande do Sul, Porto Alegre - RS. Brasil.

E-mail: anacarolrs@hotmail.com

\section{Maria Luiza Leal Pacheco}

Mestre em Psicologia Clínica pela Pontifícia Universidade Católica do Rio Grande do Sul, Porto Alegre - RS. Brasil.

E-mail: malupacheco@bol.com.br

\section{Regina Lucia Sucupira Pedroza}

Doutora em Psicologia pela Universidade de Brasília, Brasília - DF. Docente na Universidade de Brasília, Brasília - DF. Brasil.

E-mail: rpedroza@unb.br

\section{Lucia Helena Cavasin Zabotto Pulino}

Doutorado em Filosofia pela Universidade Estadual de Campinas, Campinas - SP. Docente na Universidade de Brasília, Brasília - DF. Brasil.

E-mail: luciahelena.pulino@gmail.com

Endereço para envio de correspondência: Universidade de Brasília, Departamento de Psicologia Escolar e do Desenvolvimento (Secretaria de Pós-Graduação em Processos de Desenvolvimento Humano e Saúde/ 2 Instituto de Psicologia), Campus Darcy Ribeiro, Instituto Central de Ciências (ICC), Ala Sul, Bloco A, Térreo, Asa Norte, Brasília/DF, CEP 70910-900

\section{Recebido 28/06/2016}

Reformulação 12/08/2016

Aprovado 04/11/2016

Received 06/28/2016

Reformulated $08 / 12 / 2016$

Approved 11/04/2016

Recibido 28/06/2016

Reformulado 12/08/2016

Aceptado 04/11/2016

Como citar: Noal, D. S., Vicente, L. N. Weintraub, A. C. A. M., Fagundes, S. M. S., Cabral, K. V., Simoni, A. C. R. et al. (2016). Estratégia de saúde mental e atenção psicossocial para afetados da Boate Kiss. Psicologia: Ciência e Profissão, 36(4): 932-945. doi:10.1590/1982-3703002062016

How to cite: Noal, D. S., Vicente, L. N. Weintraub, A. C. A. M., Fagundes, S. M. S., Cabral, K. V., Simoni, A. C. R. et al. (2016). Mental health and psychosocial care strategy for affected from Kiss Nightclub. Psicologia: Ciência e Profissão, 36(4): 932-945. doi:10.1590/1982-3703002062016

Cómo citar: Noal, D. S., Vicente, L. N. Weintraub, A. C. A. M., Fagundes, S. M. S., Cabral, K. V., Simoni, A. C. R. et al. (2016). Estrategia de salud mental y atención psicosocial para afectados de la Discoteca Kiss. Psicologia: Ciência e Profissão, 36(4): 932-945. doi:10.1590/1982-3703002062016 